\title{
Polar nanoregions and diffuse scattering in the relaxor ferroelectric $\mathrm{PbMg}_{1 / 3} \mathrm{Nb}_{2 / 3} \mathrm{O}_{3}$
}

\author{
M. Paściak* and T. R. Welberry ${ }^{\dagger}$ \\ Research School of Chemistry, Australian National University, Canberra ACT 0200, Australia
}

J. Kulda

Institut Laue-Langevin, BP 156, 38042 Grenoble Cedex 9, France

\author{
M. Kempa and J. Hlinka \\ Institute of Physics, Academy of Sciences of the Czech Republic, Na Slovance 2, Praha 8, Czech Republic
}

(Received 2 March 2012; published 19 June 2012)

\begin{abstract}
We present a fully atomistic model of polar nanoregions (PNRs) in the relaxor ferroelectric $\mathrm{PbMg}_{1 / 3} \mathrm{Nb}_{2 / 3} \mathrm{O}_{3}$. Our molecular dynamics calculations reproduce both the characteristic form of the neutron diffuse scattering distribution and its temperature dependence. A shell model was used with a modified version of a published interatomic potential that was based on ab initio calculations. The parameters of this potential were optimized for the present work to provide a better description of the $\mathrm{O}$ atom interactions, as these are particularly important for neutron scattering. At high temperatures the $\mathrm{Pb}$ ions are displaced from their mean site positions in a direction that has an isotropic distribution, but at low temperatures the distribution condenses into eight localized sites displaced from the average position along each of the eight possible $\left\langle\begin{array}{llll}1 & 1 & 1\end{array}\right\rangle$ directions. At intermediate temperatures $(300 \mathrm{~K})$ the distribution is cuboidal with some preference for $\langle 111\rangle$ displacements but with all possible displacement directions present. Longitudinal correlations between the displacements of $\mathrm{Pb}-\mathrm{Mg} / \mathrm{Nb}$ and $\mathrm{Pb}-\mathrm{O}$ increase monotonically in magnitude as the temperature changes from $700 \mathrm{~K}$ to $10 \mathrm{~K}$ with the sign of the $\mathrm{Pb}-\mathrm{O}$ correlation being negative. At low temperatures this increase in correlation results in polar nanoregions that are clearly visible in plots of the local structure, although the exact form of these domains is more difficult to visualize. We show that the form of these PNRs can be revealed by an examination of conditional displacement distributions at low temperatures. Therein a strongly anisotropic cooperative displacement behavior is found. Remnants of this correlation pattern persist at much higher temperatures, but progressively a relatively smaller proportion of the $\mathrm{Pb}$ ions appears to be taking part and there is a substantially larger random component. It seems likely that the onset of the characteristic structured diffuse scattering at around $400 \mathrm{~K}$ coincides with the appearance of this cooperative displacement behavior.
\end{abstract}

DOI: 10.1103/PhysRevB.85.224109

PACS number(s): 77.80.-e

\section{INTRODUCTION}

Lead-based relaxor ferroelectrics, with the classical examples being $\mathrm{PbMg}_{1 / 3} \mathrm{Nb}_{2 / 3} \mathrm{O}_{3}(\mathrm{PMN}), \mathrm{PbZn}_{1 / 3} \mathrm{Nb}_{2 / 3} \mathrm{O}_{3}(\mathrm{PZN})$, and even more so their solid solutions with $\mathrm{PbTiO}_{3}(\mathrm{PT})$, are renowned for their excellent electromechanical properties. ${ }^{1}$ At the same time the complexity of the structure-property relationship in relaxors has been stimulating fundamental research for the last few decades. ${ }^{2,3}$ In contrast to a normal ferroelectric, for which the transition to the polar phase is marked by a sharp change of dielectric constant, relaxors (RFEs) display a broad, frequency-dependent anomaly. It is now widely believed that this unusual feature is related to the existence of polar clusters [or polar nanoregions (PNRs)]. The nature of PNRs - their morphology and dynamics - has been the subject of intense debate in recent years, but so far this has not yielded a definitive nanoscale picture of relaxors.

PMN is probably the most studied relaxor compound. ${ }^{4,5}$ Macroscopically, it stays cubic (space group $P m \overline{3} m$ ) down to $5 \mathrm{~K}^{6}{ }^{6}$ There is, however, strong evidence for the structure on a local scale to differ from cubic symmetry. The first evidence of the existence of PNRs in PMN below the so-called Burns temperature, $T_{d}=620 \mathrm{~K}$, came from a refraction experiment by Burns and $\mathrm{Dacol}^{7}$ and was later confirmed by many other studies. ${ }^{8,9}$ However, very few experimental methods can observe PNRs directly. Imaging via TEM has been troublesome due to PNRs being unstable under the electron beam. The recently developed piezoresponse force microscopy (PFM) technology is useful for studying mesoscale features of relaxors. ${ }^{10}$ However, such PFM studies have shown labyrinthine patterns similar to those for classical $180^{\circ}$ ferroelectric domain structures. This is interpreted in terms of surface-induced local symmetry breaking within an otherwise cubic structure. ${ }^{10,11}$ Thus, a nanoscale picture of PNRs in PMN is not revealed.

Diffuse scattering (DS) experiments can provide direct access to local structural information in a material. ${ }^{12}$ It enables extraction of details concerning the correlations between atoms over the length scale of a few nanometers. However, as is the case for all diffraction experiments, because the intensity is the Fourier transform of the pair-distribution function only, its interpretation is subject to ambiguity. Over the last (few) years there have been many reported observations of the DS in lead-based relaxors. ${ }^{13-23}$ The polar nature of correlations giving rise to DS is now widely accepted. It is also known, however, that the expected main component of PNRs, i.e., the frozen TO soft mode, cannot alone explain the distribution of DS intensities. ${ }^{14}$ Consequently, TA-TO coupling schemes have been proposed to account for this discrepancy. ${ }^{16,24,25}$ Recent work by Bosak et al., ${ }^{26}$ in which $\mathrm{x}$-ray data are interpreted using thermal diffuse scattering formalism, further indicates 
the importance of the acoustic mode. In this light, neutron DS appears particularly important for studying relaxors, as unlike $\mathrm{x}$ rays it offers a cross section for oxygen comparable to those for the metal ions, being thus much more sensitive to polar distortions.

There have been a number of attempts to utilize computer simulations to address the question of nanoscale structure in relaxor ferroelectrics. Two quite different approaches have been followed. In one approach Welberry et al., ${ }^{27-30}$ Pasciak et al., ${ }^{31}$ and Baba-Kishi and Pasciak ${ }^{32}$ developed models that used effective atom-atom interactions in order to try to reproduce the observed DS distributions. In these models the displacements (both magnitude and direction) of individual ions away from their average site positions were defined at the outset and the interactions were used to induce correlations between these ion displacements. In the second approach, $a b$ initio-based effective-Hamiltonian simulations have been used to explain the impact of short-range chemical order on the coherence of the ferroelectric mode ${ }^{33,34}$ and, more recently, the characteristic shape of the DS. ${ }^{35}$

Although the first approach leads to models that give DS patterns that agree well with observation, the results are not entirely satisfactory, as the interactions are based on intuitive understanding of the system and not on rigorous ab initio calculations. Moreover, this work has also shown that a range of different models can lead to very similar results. ${ }^{29}$ In contrast, the second $a b$ initio-based effectiveHamiltonian approach has by principle a physically more realistic grounding, but by reducing the degrees of freedom to concentrate on selected ferroelectric modes, a fully atomistic picture of relaxors is missing. Also, in the recent attempt to obtain the characteristic shape of the DS (Ref. 35) using this approach, the quality of the agreement with the observed scattering is not satisfactory.

In an attempt to bridge the gulf between these two contrasting approaches, we report here on a study in which we have carried out molecular dynamics (MD) simulations using a shell model. ${ }^{36}$ Within such a core-shell representation, the polar nature of individual ions occurs naturally as the result of the interatomic interactions and no preset ionic shifts are assumed, as was the case in previous work. By using a model for the interactions ${ }^{37}$ that is based on ab initio calculations and which, after an optimization, reproduces fundamental experimental properties of PMN, we avoid any ambiguity. In the MD simulations all atomic positions are independent dynamic variables, allowing us to study the morphology of any PNRs and the temperature dependence of the atomic displacement correlations.

\section{DIFFUSE SCATTERING EXPERIMENT}

The neutron diffuse scattering data were collected using the FlatCone multianalyzer on the IN20 thermal neutron tripleaxis spectrometer at the Institut Laue-Langevin (ILL) high-flux reactor. This setup operates with a fixed energy of scattered neutrons, $E_{f}=18 \mathrm{meV}$ corresponding to $k_{f}=3.0 \AA^{-1}$, and both the monochromator and the analyzer crystals use (111) reflections from elastically bent silicon crystals. The elasticline energy resolution is about $1 \mathrm{meV}$, and the momentum resolution is defined by natural collimation, roughly equivalent

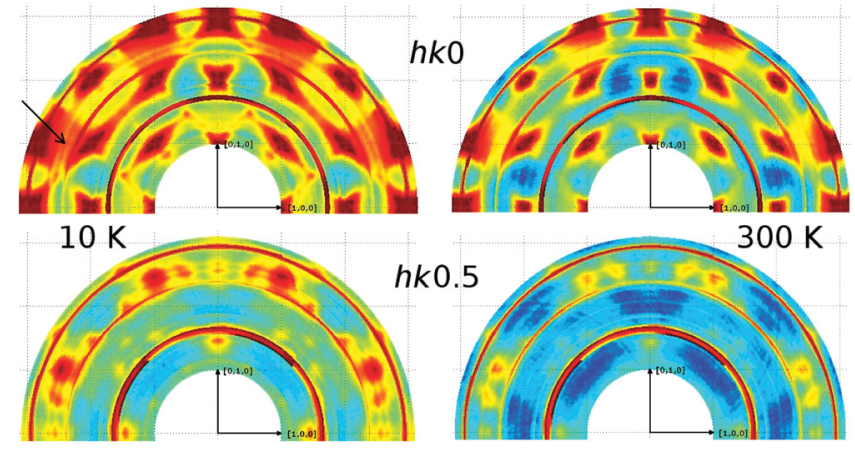

FIG. 1. (Color online) Elastic ( $\Delta E \sim 1 \mathrm{meV})$ neutron diffuse scattering. $h k 0$ (top) and $h k 0.5$ (bottom) sections at $300 \mathrm{~K}$ (right) and $10 \mathrm{~K}$ (left) are presented. The scattering intensity is displayed in a logarithmic scale. Powder rings come from $\mathrm{Nb}$ foil that was used to wrap the crystal. The arrow marks a region of possibly high intensity that is obscured by the powder scattering.

to the resolution obtained with $40^{\prime}-60^{\prime}$ Soller collimators and mosaic crystals.

The experiment was carried out with a $7 \times 7 \times 7 \mathrm{~mm}^{3}$ specimen cut from a pure PMN single crystal grown by a modified Bridgeman technique ${ }^{38}$ at Shanghai Institute of Ceramics. Specimens from the same or a similar single crystal were used also in Refs. 39-41.

The sample was wrapped in a thin $\mathrm{Nb}$ foil and mounted in a cryofurnace, permitting temperatures in the range between 2 and $490 \mathrm{~K}$ to be reached, with temperature stability of better than $0.1 \mathrm{~K}$. The sample was oriented with its [ $\left[\begin{array}{ll}0 & 0\end{array}\right]$ cubic axis vertical so that, using the flat-cone geometry, we could access, in addition to the equatorial $h k 0$ plane, vertically offset $h k l$ planes up to $l=0.5$.

Out of a larger mass of data, here we concentrate on elastic scattering on the $h k 0$ and $h k \frac{1}{2}$ planes, measured at 10 and $300 \mathrm{~K}$ for momentum transfer magnitudes between 1.5 and $4.6 \AA^{-1}$ (Fig. 1). At both temperatures the data is dominated by ridges of diffuse scattering parallel to the $\langle 110\rangle$ directions, observed and reported by many authors in the past decade. The wide angular acceptance of the FlatCone multianalyzer and its ability to navigate out of the equatorial plane allows a more complete picture of the DS to be obtained and enables the intensities in various Brillouin zones to be compared. This allows assessment of selection rules that are governed by both the directions of atomic displacements and the phase of interference between different lattice sites. The predominantly displacive origin of the DS is attested to by a general increase of its intensity with increasing momentum transfer (note that for pure phonon scattering the intensity is proportional to $Q^{2}$ ). While the elastic DS exhibits essentially the same topology at both temperatures, its intensity is much stronger at $10 \mathrm{~K}$.

At low temperatures, in agreement with some earlier x-ray observations, ${ }^{42}$ diffuse superlattice spots (e.g., $\frac{3}{2} \frac{1}{2} 0$ ) become visible, accompanied by more of such spots in the $h k \frac{1}{2}$ plane. The absence of this signal at the $h+\frac{1}{2} k+\frac{1}{2} 0$ positions, and more generally, the absence of the diffuse streaks on the $\left\langle\begin{array}{lll}1 & 1 & 0\end{array}\right.$ lines passing through the origin of the reciprocal space, indicates the predominantly transverse character of atomic 
displacement patterns. This feature has been observed already in the pioneering work of You and Zhang, ${ }^{15}$ but here it gets added support as it is based on observations over a larger portion of the reciprocal space. The more intriguing feature is the zigzag layout of such spots in the $h k \frac{1}{2}$ maps, where alternately the position of the spot is either on the inside or the outside of the exact $h$ or $k=\frac{1}{2}, \frac{3}{2}$, or $\frac{5}{2}$ lines. This asymmetry leads to speculation about the possible involvement of local size-effect modulations.

In addition, the $h k \frac{1}{2}$ maps reveal the presence of diffuse superlattice spots at the $h+\frac{1}{2} k+\frac{1}{2} \frac{1}{2}$ positions. Again, there seems to be a general tendency of increasing intensity with increasing momentum transfer, indicating that at least in part the contrast is caused by atomic displacements. However, there is a significantly weaker temperature dependence and their persistence up to the highest temperatures indicates that these spots have their origins in the chemical short-range order.

\section{III. $\mathrm{Mg} / \mathrm{Nb}$ DISTRIBUTION}

B-site chemical ordering has been a key feature in many PMN studies described in the literature. It is well known that in some $\mathrm{PbB}_{1 / 2}^{\prime} \mathrm{B}_{1 / 2}^{\prime \prime} \mathrm{O}_{3}$ systems, e.g., $\mathrm{PbSc}_{1 / 2} \mathrm{Ta}_{1 / 2} \mathrm{O}_{3}$, the degree of chemical ordering can change ferroelectric properties from normal (anti)ferroelectric in ordered crystals to completely relaxor in disordered ones. ${ }^{2,43}$ However, in the case of PMN, it has been found that engineering the chemical ordering is not viable; B-site distribution does not change upon annealing. This fact led to the so-called space-charge model in which domains with $1: 1 \mathrm{Mg} / \mathrm{Nb}$ order were immersed in an $\mathrm{Nb}$-rich matrix. Later studies have shown that a preparation of ordered samples is possible with a small amount of doping (e.g., with La) and that the random-site model provides a better representation of ordered chemical domains in PMN. ${ }^{44,45}$ However, diffraction studies ${ }^{13,46}$ show very weak superstructure $\frac{1}{2}(111)$ peaks from which one can conclude that normally a relatively small proportion of the whole crystal is ordered. To study the whole spectrum of possible arrangements of atoms, Bursill et al. ${ }^{47,48}$ developed an extended next-nearest-neighbor Ising (eNNNI) model in which the growth of the $\mathrm{NaCl}$-type ordered $\mathrm{Mg} / \mathrm{Nb}$ domains is limited by an electrostatic (space-charge) term. A Monte Carlo simulation with this model leads to $1: 1$ ordered domains of a size up to $50 \AA$, but there is no two-phase $\left(\mathrm{PbMg}_{1 / 2} \mathrm{Nb}_{1 / 2} \mathrm{O}_{3}\right.$ and $\mathrm{PNbO}_{3}$ ) segregation.

In a study of PZN by Welberry et al. ${ }^{28} \mathrm{NaCl}$-type ordering of the B-site ions was created using a simple nearest-neighborIsing (NNI) model. The 2:1 stoichiometry limits the degree of order that can be induced. Furthermore, since in this model (unlike in the NNNI model) there is no energy gain from the next nearest neighbors being the same, large 1:1 chemical domains are not favored. The alternation of $\mathrm{Zn}$ and $\mathrm{Nb}$ along the $\left\langle\begin{array}{lll}1 & 0 & 0\end{array}\right\rangle$ direction exists only over a short range, but $\mathrm{Zn}$ is almost always succeeded by $\mathrm{Nb}$, while $\mathrm{Nb}$ may be followed by either $\mathrm{Nb}$ or $\mathrm{Zn}$.

In the present study we use the same methodology, i.e., a Monte Carlo (MC) simulation with the simple NNI model, to derive a B-site occupancy distribution for $\mathrm{Mg}$ and $\mathrm{Nb}$. The $\mathrm{MC}$ energy has the form

$$
E_{\mathrm{B} \text {-site }}=\sum_{\substack{\text { All }\langle 100\rangle \\ \text { vectors }}} J \sigma_{m} \sigma_{n} .
$$

Here $\sigma_{m}$ and $\sigma_{n}$ are $(+1 /-1)$ Ising spin variables at sites $m$ and $n$, respectively, on the primitive cubic B-site lattice. We adjust the value of $J / k T$ to produce a structure in which the degree of ordering is lower than in PZN. This choice is made because of the observation that in our PMN sample, similar to previous diffraction studies, ${ }^{13,46}$ the peaks due to occupancy order that occur in the $h k 0.5$ section are significantly more diffuse than those reported for PZN. During MC simulation only nearest-neighbor atoms were allowed to swap positions, preserving local charge balance.

The distribution of B-site ions is established on formation of the crystal and can only change via diffusional processes. It must therefore be assumed to remain unchanged on the time scales in which the PNR dynamical processes (that we seek to investigate using MD) will occur. Therefore the $\mathrm{Mg} / \mathrm{Nb}$ arrangements that were created using the MC procedure for different system sizes were held constant throughout all of the subsequent simulations. Figure 5(c) shows a single layer of the representative B-site distribution in the largest system studied. Dark (red online) squares represent unit cells containing $\mathrm{Mg}$ and white squares unit cells containing $\mathrm{Nb}$. The structure contains small 1:1 domains (up to $40 \AA$ in size) and there is no phase separation. In this respect our chemical distribution is very similar to the ones created by Bursill et al. ${ }^{47}$

It should be noted that in the MD simulations presented below, electrostatics is included explicitly, so avoiding speculations on the strength of the electric random field that in effective Hamiltonian studies is often adjusted arbitrarily.

\section{MOLECULAR DYNAMICS SIMULATIONS}

For the molecular dynamics simulations we used a pair potential based on that developed specifically for PMN by Sepliarsky et al. ${ }^{49}$ and Sepliarsky and Cohen. ${ }^{37}$ This is a shell pair potential, the parameters for which were based on ab initio [density functional theory (DFT)] calculations. Within the model each atom is represented by a core and a shell. There are electrostatic interactions between all cores and shells, except for those within the same atom. For these an anharmonic spring of the form

$$
V^{c-s}(r)=k_{2} r^{2}+k_{4} r^{4}
$$

is used, where $r$ is a distance between the core and shell. Short-range interactions are represented by a Rydberg form,

$$
V(r)=(a+b r) \exp (-r / c),
$$

where $r$ is a distance between two atoms. This shell potential has been used in a study of phase stability of PMN-PT (Ref. 37) and elastic properties of PMN. ${ }^{50}$ Our preliminary simulations ${ }^{12}$ using this potential demonstrated that the model reasonably well reproduces cation correlations and the calculated x-ray DS patterns qualitatively agree with experimental ones.

However, due to the well-known difficulty for DFT localdensity approximation (LDA) calculations to converge to 
TABLE I. Refined parameters for the interatomic potential.

\begin{tabular}{|c|c|c|c|c|}
\hline \multicolumn{5}{|c|}{ Short-range parameters } \\
\hline Atomic pair / parameter & $a$ & $b$ & $c$ & \\
\hline $\mathrm{Pb}-\mathrm{O}$ & 6105.11 & 20.52 & 0.2677 & \\
\hline $\mathrm{Mg}-\mathrm{O}$ & 972.91 & 0.22 & 0.3002 & \\
\hline $\mathrm{Nb}-\mathrm{O}$ & 1550.93 & 1.35 & 0.3024 & \\
\hline $\mathrm{O}-\mathrm{O}$ & 519.40 & -102.67 & 0.3099 & \\
\hline \multicolumn{5}{|c|}{ Core-shell parameters } \\
\hline Atom/parameter & Core charge & Shell charge & $k_{2}$ & $k_{4}$ \\
\hline $\mathrm{Pb}$ & 4.5039 & -2.8131 & 46.76 & 26697.10 \\
\hline $\mathrm{Mg}$ & 2.1353 & -0.0321 & 142.33 & 0.0 \\
\hline $\mathrm{Nb}$ & 6.3954 & -2.9623 & 447.62 & 254.13 \\
\hline $\mathrm{O}$ & 0.4988 & -2.0590 & 8.64 & 1944.40 \\
\hline
\end{tabular}

experimental lattice constants, the unit cell obtained in the above simulations was rather small. One consequence of this was that the oxygen atoms appeared to be overcorrelated. Since oxygen scattering is relatively much more important for neutrons than for $\mathrm{x}$ rays, this results in a poor agreement of experimental and calculated neutron DS. Consequently, for the present study we have further optimized the parameters of this potential in order to obtain a better agreement between experimental and calculated properties. The optimization cost function included lattice parameters, atomic displacement parameters, bulk and shear moduli, elastic constants, the high-frequency dielectric constant $\left(\varepsilon_{\infty}\right)$, and Born effective charges. For the mean-square displacements the experimental values used for comparison were based on the average structure parameters of Bonneau et al. ${ }^{51}$ Average values were used without specifying particular displacement directions in order to avoid building into the optimized potential any directionality that could unduly influence the form of the polar nanoregions that might develop. Bulk and shear moduli as well as elastic constants were taken from the work of Ahart et al. ${ }^{50}$ As core-shell parameters were also altered, we took into account Born effective charges and $\varepsilon_{\infty}$ given by Prosandeev et al. ${ }^{52}$ The charges were given relatively small weights to account for the fact that they had not been obtained experimentally.

On the assumption that the initial set of parameters falls close to the minimum of the cost function, a reverse Monte Carlo (RMC) procedure was set up to explore parameter space. At the early stages of the refinement the optimization cost function also included the global instability index ${ }^{53}$ that allowed us to quickly exclude unstable solutions. Each RMC step comprised MD equilibration runs with a relatively small system of $6^{3}$ unit cells at three temperatures $(600 \mathrm{~K}, 300 \mathrm{~K}$, and $10 \mathrm{~K}$ ). The program GULP (Ref. 54) was used to calculate effective charges $\varepsilon_{\infty}$ and elastic properties. Refined parameters are presented in Table I.

We have used the program DLPOLY (Ref. 55) and a constant stress, constant temperature $(\mathrm{N} \sigma \mathrm{T})$ ensemble for all molecular dynamics simulations. For the simulations with the ultimate potential form, large $(48 \times 48 \times 48$ unit cells $)$ systems were used to enable reasonable resolution of calculated diffraction patterns as well as unconstrained development of polar clusters. We used a 0.5 -fs time step and the typical number of steps was $10^{5}$. DS patterns were obtained with the program DISCUS. ${ }^{56}$ Each reciprocal space map is an average over ten
DS patterns for instantaneous structures recorded at different times of the same simulation run.

\section{RESULTS}

The average distribution of atoms in the perovskite unit cell of the MD simulations of PMN carried out at 10 and

(a)

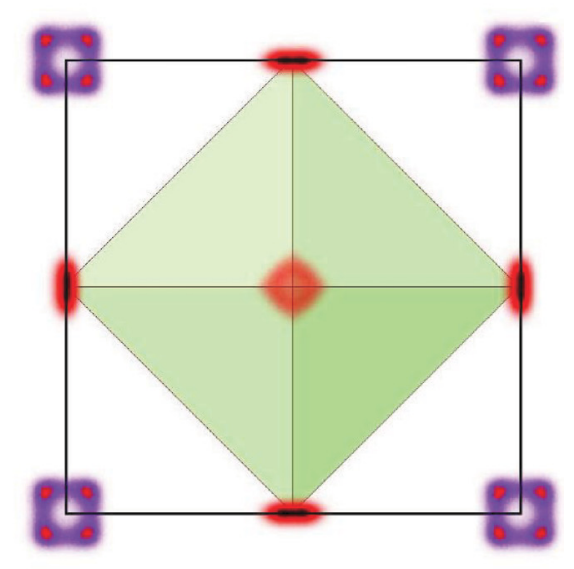

(b)

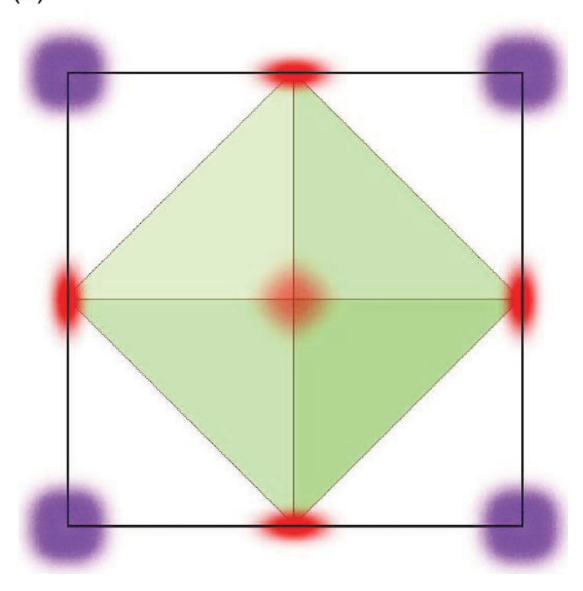

FIG. 2. (Color online) Distribution of lead and oxygen atoms within a perovskite unit cell from MD simulation at (a) $10 \mathrm{~K}$ and (b) $300 \mathrm{~K}$. 
TABLE II. Comparison of experimental (in parentheses) and calculated PMN properties. Lattice and displacement parameter data from Ref. 51, bulk modulus data from Ref. 50.

\begin{tabular}{lccc}
\hline \hline Temperature & $10 \mathrm{~K}$ & $300 \mathrm{~K}$ & $600 \mathrm{~K}$ \\
\hline Lattice parameter $(\AA)$ & $4.043(4.050)$ & $4.051(4.050)$ & $4.063(4.057)$ \\
Bulk modulus $(\mathrm{GPa})$ & $118(103)$ & $114(95)$ & $128(113)$ \\
$\Delta \mathrm{Pb}(\AA)$ & $0.289(0.331)$ & $0.306(0.354)$ & $0.332(0.361)$ \\
$\Delta \mathrm{Mg} / \Delta \mathrm{Nb}(\AA)$ & $0.064(0.110)$ & $0.110(0.134)$ & $0.145(0.180)$ \\
$\Delta \mathrm{O}^{\perp}(\AA)$ & $0.206(0.220)$ & $0.220(0.271)$ & $0.242(0.308)$ \\
$\Delta \mathrm{O}^{\|}(\AA)$ & $0.056(0.075)$ & $0.072(0.103)$ & $0.087(0.120)$ \\
\hline \hline
\end{tabular}

$300 \mathrm{~K}$ are shown in Fig. 2. The disorder of $\mathrm{Pb}$ and $\mathrm{O}$ atoms is clearly revealed by the shapes of the atomic distributions. At $300 \mathrm{~K}$ the shapes are cuboidal for $\mathrm{Pb}$ and approximately ellipsoidal for $\mathrm{O}$, corresponding well to the structure reported by Bonneau et al. ${ }^{51}$ At $10 \mathrm{~K}$ the cuboidal $\mathrm{Pb}$ distribution clearly resolves into eight discrete peaks displaced from the center along each of the $\left\langle\begin{array}{llll}1 & 1 & 1\end{array}\right\rangle$ directions. That at low temperatures $\left\langle\begin{array}{lll}1 & 1 & 1\rangle \\ \text { directions of polarization dominate is in agreement }\end{array}\right.$ with the results of a pair distribution function (PDF) study. ${ }^{9}$ [Note that the distributions plotted in Fig. 2 are for the atomic cores only.] In the case of the $\mathrm{O}$ atoms the shell positions closely follow the core positions, but for the $\mathrm{Pb}$ atoms the shell positions tend to be somewhat more confined to the center of the atomic site. This can be interpreted in terms of the core-shell representation of the $\mathrm{Pb}$ electron lone pair.

In addition to the shapes of the atomic distributions shown in Fig. 2, the potential reproduces well other structural measures of PMN (see Table II for details). Lattice parameters at all three temperatures are reproduced within $0.25 \%$. The bulk modulus was obtained using the lattice property software (GULP) ${ }^{54}$ $\Delta \mathrm{Pb}, \Delta \mathrm{Mg} / \Delta \mathrm{Nb}, \Delta \mathrm{O}^{\perp}$, and $\Delta \mathrm{O}^{\|}$are rms displacement parameters. $\Delta \mathrm{O}^{\perp}$ and $\Delta \mathrm{O}^{\|}$correspond to displacements of $\mathrm{O}$ atoms perpendicular and parallel to the $\mathrm{B}-\mathrm{O}$ bonds, respectively.

Figure 3 shows neutron DS patterns calculated from the MD simulations at 10 and $300 \mathrm{~K}$ for the $h k 0$ and $h k 0.5$ sections (corresponding to the experimental measurements shown in Fig. 1). The well-known features of relaxor DS patterns are all well reproduced. This is quite remarkable given the potential fitting procedure did not take into account any

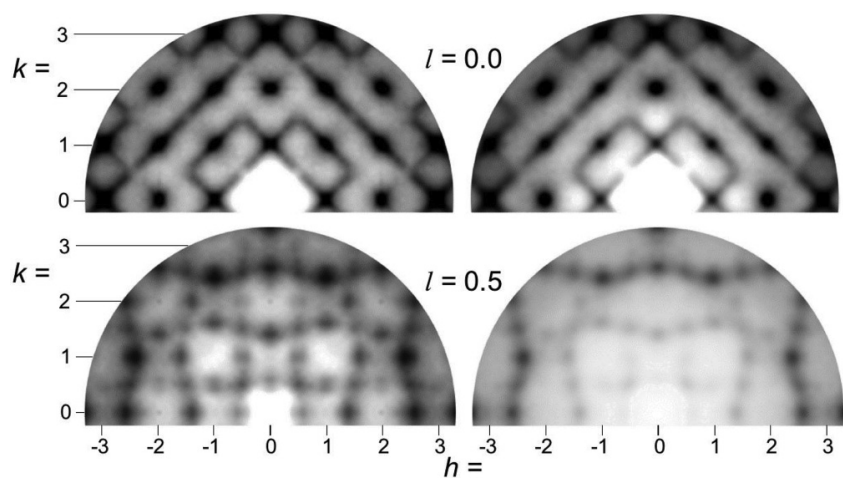

FIG. 3. Calculated neutron diffuse scattering (logarithmic scale) at two temperatures $300 \mathrm{~K}$ (right) and $10 \mathrm{~K}$ (left) and on two layers of the reciprocal space, $h k 0$ (top) and $h k 0.5$ (bottom). quantity directly related to DS. The various diffuse features include the diffuse rods of scattering running along $\left\{\begin{array}{lll}1 & 1 & 0\end{array}\right\}$ reciprocal rows. These intensify around Bragg positions and where two rods intersect characteristic butterfly-shaped diffuse peaks are formed. The overall azimuthal variation of intensity in the rods is such that those passing through the origin of reciprocal space have zero intensity. In the $h k 0.5$ section, these rods give rise to diffuse peaks that appear near positions $\left(n, m+\frac{1}{2}, \frac{1}{2}\right)$ and $\left(n+\frac{1}{2}, m, \frac{1}{2}\right)$, with $n, m$ integral. Another set of diffuse peaks in this section at positions $\left(n+\frac{1}{2}, m+\frac{1}{2}, \frac{1}{2}\right)$ is derived from the B-site ordering. It should also be mentioned that these peaks do not derive solely from the occupational short-range order of the B-site cations but are enhanced by the associated ionic displacements. This is supported by the fact that no temperature-independent spots can be observed and by the fact that the peaks close to the origin are weak while those at higher $Q$ are more intense. A comparable situation was found in cubic-stabilized zirconia where the effect of the basic oxygen vacancy distribution was not observed directly but only indirectly via the resulting cation relaxations. ${ }^{57}$

Another point to note regarding the diffuse peaks that appear in the $h k 0.5$ sections of Fig. 3 is that they form vertical and horizontal rows that have a zigzag character to them. This matches the positions of the corresponding peaks in the observed data (Fig. 1), though the effect is rather masked by the powder ring artifacts. The shift of the individual intensity maxima from the exact $\left(n, m+\frac{1}{2}, \frac{1}{2}\right)$ or $\left(n+\frac{1}{2}, m, \frac{1}{2}\right)$ positions must be related to a change of the structure factor at the Brillouin zone edge. Such asymmetric DS distribution across a zone edge is characteristic of the size effect (local distortion of a lattice due to different species occupying the same crystallographic position). ${ }^{58}$ Thus the zigzag pattern may be considered to arise from the dissimilar environments of $\mathrm{Mg}$ and $\mathrm{Nb}$ atoms but is not directly related to the degree of the B-site ordering.

To understand the mechanisms that lead to particular local structure arrangements and to the appearance of DS, we performed MD simulations in a wide range of temperatures (700-10 K). Different structural parameters characterizing a smeared transition state in PMN are presented in Fig. 4.

In agreement with experimental results, ${ }^{15}$ the intensity of DS around the (300) Bragg reflection increases with decreasing temperature. In Fig. 4(a) the ratio of DS intensities around (300) and (200) is presented with a clear change around $400 \mathrm{~K}$. This behavior closely resembles that measured by Hiraka et al. ${ }^{8}$ and Gehring et al., ${ }^{4}$ where $T_{d}=420 \mathrm{~K}$ has been found to be the new estimation of Burns temperature at which polar clusters become static. ${ }^{59}$ To relate the changes of DS to polar and acoustic modes, we calculate the correlation of atomic displacements [Fig. 4(b)] as

$$
\frac{1}{12 N_{\mathrm{Pb}}} \sum_{\langle i, j\rangle} \hat{\boldsymbol{\xi}}_{i}^{\mathrm{Pb}} \cdot \hat{\boldsymbol{\xi}}_{j}^{O} \text { and } \frac{1}{8 N_{\mathrm{Pb}}} \sum_{\langle i, j\rangle} \hat{\boldsymbol{\xi}}_{i}^{\mathrm{Pb}} \cdot \hat{\boldsymbol{\xi}}_{j}^{\mathrm{Nb} / \mathrm{Mg}}
$$

for $\mathrm{Pb}-\mathrm{O}$ and $\mathrm{Pb}-\mathrm{B}$ site pairs, respectively. The sums run over nearest-neighboring pairs only, and $\hat{\boldsymbol{\xi}}_{i}$ is a unit vector in the direction of the instantaneous displacement from the averagelattice position at the $i$ th site.

The high-temperature value of $\mathrm{Pb}-\mathrm{O}_{12}$ correlations (ca. -0.3$)$ results from low-frequency TO mode vibrations 


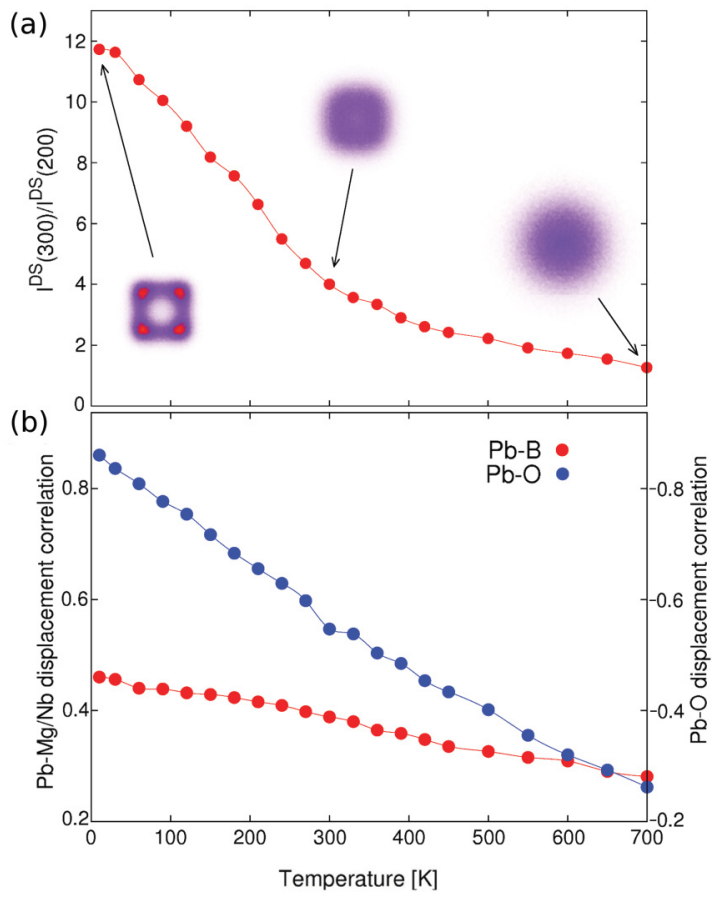

FIG. 4. (Color online) (a) Temperature dependence of the ratio of the diffuse scattering intensity integrated from the areas at $(3 \pm 0.1$ $0 \pm 0.10)$ and $(2 \pm 0.10 \pm 0.10)$. Insets show the distribution of $\mathrm{Pb}$ atoms at 700,300 , and $10 \mathrm{~K}$. (b) Temperature dependence of $\mathrm{Pb}-\mathrm{O}_{12}$ and $\mathrm{Pb}-\mathrm{B}_{8}$ equal-time correlations. See text for details.

(so called Last mode ${ }^{60}$ ). Clearly, already at $700 \mathrm{~K}$ there exist dynamic polar entities, in agreement with a recent NMR study. ${ }^{61}$ The anticorrelation increases with cooling (to reach the value of -0.84 at $10 \mathrm{~K}$ ), leading to the appearance of more and more structured dipole patterns (Fig. 5). The correlation of cation displacements (Pb-Mg/Nb, Fig. 4(b) also steadily increases with cooling, although at a somewhat slower pace. This confirms an atomistic picture of local structure of PMN, where TO mode softening is coupled to correlations of a rather acoustic type. ${ }^{15,25} \mathrm{~Pb}$-B cation correlation can be also understood in terms of bond valence rationale $-\mathrm{Pb}$ and $\mathrm{Nb}$ atoms will tend to move in concert to satisfy their valence requirements.

Figures 5(a) and 5(b) show plots of the distribution of polarization vectors for each unit cell in a single layer normal to the $\left[\begin{array}{ll}10 & 0\end{array}\right]$ direction of the MD simulations at 300 and $10 \mathrm{~K}$, respectively. From these it is difficult to discern any polarized domains at $300 \mathrm{~K}$, while at $10 \mathrm{~K}$ domains are clearly visible, although this is just a single layer and the three-dimensional nature of the domains is difficult to visualize. Figure 5(c) shows the distribution of the $\mathrm{Mg} / \mathrm{Nb} \mathrm{B}$-site ordering in the same region of the model. There is little evidence that the $\mathrm{B}$-site distribution has any influence on the pattern of polarized domains. There are a few instances where antiphase shifts in the alternating B-site pattern are visible (as columns of white cells extending over ten or more unit cells) in Fig. 5(c), and there is a suggestion that these may have some effect on the polar domains in Fig. 5(b).

In earlier works of both ourselves ${ }^{27,28}$ and others, ${ }^{62}$ much was made of the fact that the presence of one-dimensional (a)

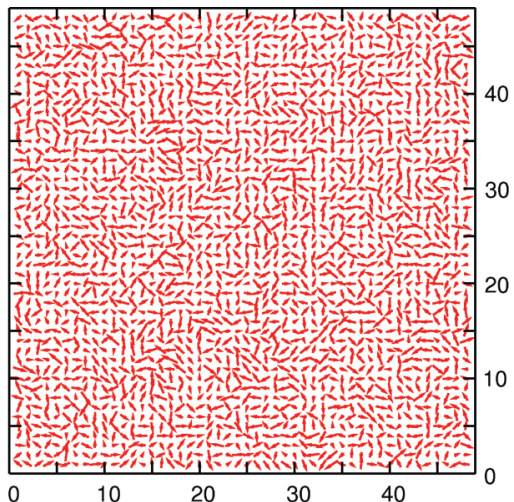

(b)

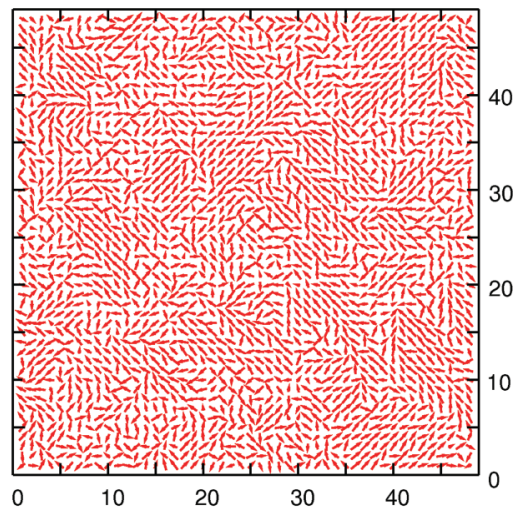

(c)

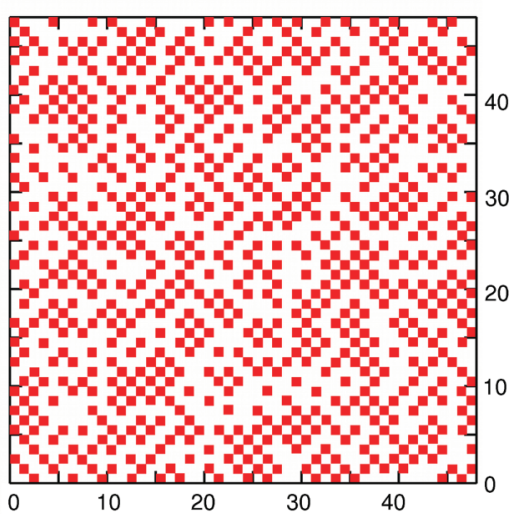

FIG. 5. (Color online) Local instantaneous dipole structure at $300 \mathrm{~K}$ (a) and $10 \mathrm{~K}$ (b) on an $x y$ plane. Each arrow represents a dipole moment of an A-site-centered single-unit cell; face- and corner-located atomic displacements are taken with an appropriate weight. (c) B-site distribution on the same $x y$ plane: (white squares) $\mathrm{Nb}$ atoms, (red squares) $\mathrm{Mg}$ atoms.

rods of diffuse scattering along the $\{110\}$ face in reciprocal space must correspond to planar domains normal to the $\langle 110\rangle$ direction in real space. Although these models were rather simplistic, they did qualitatively reproduce all the observed diffraction features and it seems unlikely that they could be completely wrong. Yet when one looks at the polar distributions shown in Fig. 5, it is difficult to see how these can possibly be reconciled with a model of planar domains as previously envisaged.

In order to obtain more insight into this problem, we plot in Fig. 6 some conditional displacement distributions that provide a better opportunity to observe such planar correlations. In 
(a)

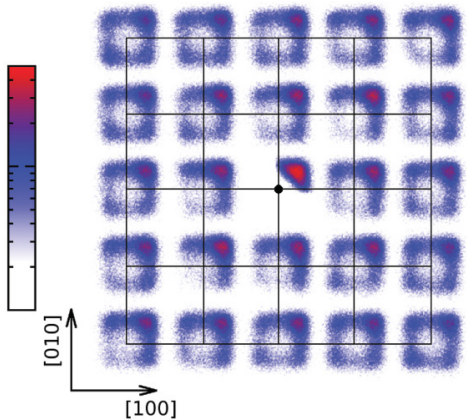

(b)

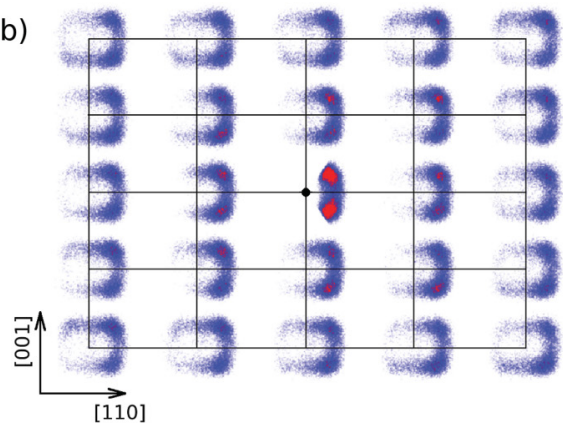

(c)

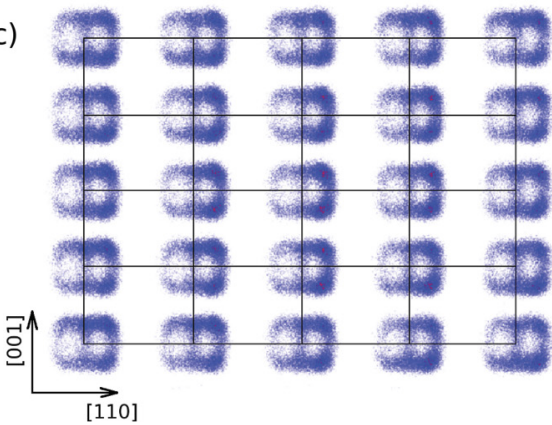

FIG. 6. (Color online) Conditional distribution of $\mathrm{Pb}$ atomic displacements at $10 \mathrm{~K}$. Plots show the displacements' distribution on $\mathrm{Pb}$ sites (scaled $\times 5$ ) in the vicinity of any $\mathrm{Pb}$ atom that has a big component of the displacement along the [110] direction. (a) (001) layer, (b) and (c) (1 110$)$ layers, containing the central atom and one-unit cell below the central atom, respectively.

Fig. 6(a) we plot for the 10-K MD results the positions of the $\mathrm{Pb}$ atoms in the unit cells surrounding a central cell in which the $\mathrm{Pb}$ displacement has a strong [1 110$]$ component. That is, from the average distribution shown in Fig. 2 we use only one quarter of the total sites-those containing $\mathrm{Pb}$ shifts along the $\left[\begin{array}{lll}1 & 1 & 1\end{array}\right]$ or $\left[\begin{array}{lll}1 & 1 & \overline{1}\end{array}\right]$ direction. It is clearly seen that the $\mathrm{Pb}$ displacements in the surrounding sites are correlated to that in the central site. Moreover, the correlation persists more

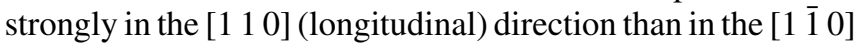
(transverse) direction.

Figure 6(b) shows a similar plot but for a single layer normal to the $\left[\begin{array}{ll}1 & 1\end{array}\right]$ direction. Now it is seen that within this layer the displacements of the $\mathrm{Pb}$ atoms in all of the surrounding sites are strongly correlated with those of the central site. This is true

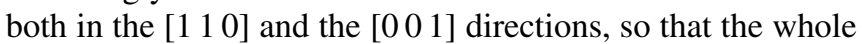
plane has concerted $\mathrm{Pb}$ displacements with a strong [1 110$]$ (on average) polarization component. Figure 6(c) shows the corresponding distributions in the layer one-unit cell below that shown in Fig. 6(b). This shows that the clear concerted

correlation seen within a single [ 110 l 10 layer has substantially diminished in layers immediately above or below the target layer.

Similar plots calculated for the $300 \mathrm{~K}$ simulation show similar behavior but the effect is much less pronounced. While there is still clear evidence for some sheetlike cooperative displacement behavior, a relatively smaller proportion of the $\mathrm{Pb}$ ions is taking part and there is a substantially larger random component. It seems likely that the onset of the characteristic structured diffuse scattering at around $400 \mathrm{~K}$ (Refs. 4 and 8) may be attributed to the appearance of this kind of cooperative displacement behavior.

\section{CONCLUSIONS}

We have presented a fully atomistic model (with all degrees of freedom taken into account) of PNRs in the relaxor ferroelectric PMN. Our MD calculations reproduce both the characteristic form of the diffuse scattering distribution and its temperature dependence. The interatomic potential used was based on a potential by Sepliarsky et al. ${ }^{37,49}$ The parameters of this potential were optimized in the present work to provide better treatment of the O-atom interactions, which are particularly important for neutron scattering. The modified potential contained no preconceived bias regarding the preferred direction of $\mathrm{Pb}$ displacements that was a feature of many early models. The present study shows that at high temperatures the $\mathrm{Pb}$ ions are displaced from their mean site positions in a direction that has an isotropic distribution, but at low temperatures the distribution condenses into eight localized sites displaced from the average position along each of the eight possible $\left\langle\begin{array}{lll}1 & 1 & 1\end{array}\right\rangle$ directions. At intermediate temperatures $(300 \mathrm{~K})$ the distribution is cuboidal with some preference for $\langle 111\rangle$ displacements but with all possible displacement directions present.

Longitudinal correlations between the displacements of $\mathrm{Pb}-\mathrm{Mg} / \mathrm{Nb}$ and $\mathrm{Pb}-\mathrm{O}$ increase monotonically in magnitude as the temperature changes from 700 to $10 \mathrm{~K}$, with the sign of the $\mathrm{Pb}-\mathrm{O}$ correlation being negative. At low temperatures this increase in correlation results in polar nanoregions that are clearly visible in plots of the local structure, though the exact form of these domains is more difficult to visualize. We have shown that by plotting conditional displacement distributions as in Fig. 6, some insight into the nature of these PNRs can be gained. In planes, normal to each of the six $\left\langle\begin{array}{lll}1 & 1 & 0\rangle\end{array}\right.$ directions, cooperative displacement behavior is found. For example, in planes normal to [1 $\overline{1} 0]$, if a particular target site is occupied

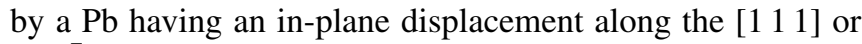
$\left[\begin{array}{lll}1 & 1 & 1\end{array}\right]$ direction, then the displacements of the $\mathrm{Pb}$ atoms in all of the surrounding sites within the plane are strongly correlated with that of this central site. This is true both in the [ $\left.\begin{array}{lll}1 & 1 & 0\end{array}\right]$ and the [001] directions so that the whole plane has concerted $\mathrm{Pb}$ displacements with a big [1 110$]$ (on average) polarization component. Moreover, this cooperative behavior is strongly anisotropic, since comparable plots of a layer one-unit cell beneath or above show lower correlation with the target site.

Remnants of this correlation pattern persist at much higher temperatures, but progressively a relatively smaller proportion of the $\mathrm{Pb}$ ions appear to be taking part and there is a substantially larger random component. It seems likely that 
the onset of the characteristic structured diffuse scattering at around $400 \mathrm{~K}$ coincides with the appearance of this anisotropic cooperative displacement behavior.

We believe that the development of the shell model potential described here and its subsequent use in MD simulations represents a significant step forward in establishing an understanding of the nature of polar nanoregions (PNRs) and the role they play in giving PMN and other relaxors their unique piezoelectric properties. The aim of the work was to try to bridge the gap between the results provided by theoretical studies of the basic interatomic interactions using $a b$ initio calculations and the results of rather heuristic approaches that have used rather simplistic models to explain the observed diffuse scattering effects. While the present work has gone a long way towards achieving this goal, it cannot be claimed that this is the last word on what is a very complex problem. The potential used was based on one developed using ab initio calculations, but it was found necessary to make subsequent adjustments to this in order to better take account of the $\mathrm{O}$ atoms that are important for neutron scattering. Although these adjustments provided a more satisfactory potential, the optimization process had a number of limitations and it is likely that more work that overcomes these will allow further improvement. One limitation was that it was necessary to use small system sizes during the optimization process in order to keep the computation time within reasonable bounds. This led to inevitable uncertainties in the derived parameters. A second limitation that similarly impacted on computation time was that only small changes to the potential parameters could be made at each step of the optimization; otherwise, the system had a tendency to become unstable. However, despite these caveats the model as described will provide a good starting point for future studies and allow exploration of many aspects of the structure and dynamics of these and similar materials.

\section{ACKNOWLEDGMENTS}

We gratefully acknowledge the support of the Australian Research Council in the form of a Discovery Project Grant. Computational time at NCF, Canberra, is also gratefully acknowledged. We thank R. E. Cohen and M. Sepliarsky for making their interatomic potential available prior to its publication. *marekp@rsc.anu.edu.au

${ }^{\dagger}$ welberry@ rsc.anu.edu.au

${ }^{1}$ S. E. Park and T. R. Shrout, J. Appl. Phys. 82, 1804 (1987).

${ }^{2}$ L. E. Cross, Ferroelectrics 76, 241 (1987).

${ }^{3}$ A. Bokov and Z.-G. Ye, J. Mater. Sci. 41, 31 (2006).

${ }^{4}$ P. M. Gehring, H. Hiraka, C. Stock, S.-H. Lee, W. Chen, Z.-G. Ye, S. B. Vakhrushev, and Z. Chowdhuri, Phys. Rev. B 79, 224109 (2009).

${ }^{5}$ A. A. Bokov, B. J. Rodriguez, X. Zhao, J.-H. Ko, S. Jesse, X. Long, W. Qu, T. H. Kim, J. D. Budai, A. N. Morozovska et al., Z. Kristallogr. 226, 99 (2010).

${ }^{6}$ N. de Mathan, E. Husson, G. Calvarn, J. R. Gavarri, A. W. Hewat, and A. Morell, J. Phys.: Condens. Matter 3, 8159 (1991).

${ }^{7}$ G. Burns and F. H. Dacol, Solid State Commun. 48, 853 (1983).

${ }^{8}$ H. Hiraka, S.-H. Lee, P. M. Gehring, G. Xu, and G. Shirane, Phys. Rev. B 70, 184105 (2004).

${ }^{9}$ I.-K. Jeong, T. W. Darling, J. K. Lee, T. Proffen, R. H. Heffner, J. S. Park, K. S. Hong, W. Dmowski, and T. Egami, Phys. Rev. Lett. 94, 147602 (2005).

${ }^{10}$ A. Kholkin, A. Morozovska, D. Kiselev, I. Bdikin, B. Rodriguez, P. Wu, A. Bokov, Z.-G. Ye, B. Dkhil, L.-Q. Chen et al., Adv. Funct. Mater. 21, 1977 (2011).

${ }^{11}$ V. V. Shvartsman and A. L. Kholkin, Z. Kristallogr. 226, 108 (2011).

${ }^{12}$ M. Paściak and T. R. Welberry, Z. Kristallogr. 226, 113 (2011).

${ }^{13}$ S. Vakhrushev, A. Nabereznov, S. K. Sinha, Y. P. Feng, and T. Egami, J. Phys. Chem. Solids 57, 1517 (1996).

${ }^{14}$ A. Naberezhnov, S. Vakhrushev, B. Dorner, D. Strauch, and H. Moudden, Eur. Phys. J. B 11, 13 (1999).

${ }^{15}$ H. You and Q. M. Zhang, Phys. Rev. Lett. 79, 3950 (1997).

${ }^{16}$ K. Hirota, Z.-G. Ye, S. Wakimoto, P. M. Gehring, and G. Shirane, Phys. Rev. B 65, 104105 (2002).

${ }^{17}$ G. Xu, G. Shirane, J. R. D. Copley, and P. M. Gehring, Phys. Rev. B 69, 064112 (2004).
${ }^{18}$ S. Vakhrushev, A. Ivanov, and J. Kulda, Phys. Chem. Chem. Phys. 7, 2340 (2005)

${ }^{19}$ J. Hlinka, S. Kamba, J. Petzelt, J. Kulda, C. A. Randall, and S. J. Zhang, J. Phys.: Condens. Matter 15, 4249 (2003).

${ }^{20}$ B. Mihailova, B. Maier, C. Paulmann, T. Malcherek, J. Ihringer, M. Gospodinov, R. Stosch, B. Güttler, and U. Bismayer, Phys. Rev. B 77, 174106 (2008).

${ }^{21}$ R. Whitfield, A. Studer, and D. Goossens, Metall. Mater. Trans. A (in press).

${ }^{22}$ R. Whitfield, D. Goossens, A. Studer, and J. Forrester, Metall. Mater. Trans. A (in press).

${ }^{23}$ K. Z. Baba-Kishi, J. Appl. Crystallogr. 44, 111 (2011).

${ }^{24}$ Y. Yamada and T. Takakura, arXiv:cond-mat/0209573.

${ }^{25}$ S. Wakimoto, C. Stock, R. J. Birgeneau, Z.-G. Ye, W. Chen, W. J. L. Buyers, P. M. Gehring, and G. Shirane, Phys. Rev. B 65, 172105 (2002).

${ }^{26}$ A. Bosak, D. Chernyshov, S. Vakhrushev, and M. Krisch, Acta Crystallogr. Sect. A 68, 117 (2012).

${ }^{27}$ T. R. Welberry, M. J. Gutmann, H. Woo, D. J. Goossens, G. Xu, C. Stock, W. Chen, and Z.-G. Ye, J. Appl. Crystallogr. 38, 639 (2005).

${ }^{28}$ T. R. Welberry, D. J. Goossens, and M. J. Gutmann, Phys. Rev. B 74, 224108 (2006).

${ }^{29}$ T. R. Welberry and D. J. Goossens, J. Appl. Crystallogr. 41, 606 (2008).

${ }^{30}$ T. R. Welberry, Metall. Mater. Trans. A 39, 3170 (2008).

${ }^{31}$ M. Paściak, M. Wołcyrz, and A. Pietraszko, Phys. Rev. B 76, 014117 (2007).

${ }^{32}$ K. Z. Baba-Kishi and M. Pasciak, J. Appl. Crystallogr. 43, 140 (2010).

${ }^{33}$ S. Tinte, B. P. Burton, E. Cockayne, and U. V. Waghmare, Phys. Rev. Lett. 97, 137601 (2006). 
${ }^{34}$ B. P. Burton, E. Cockayne, S. Tinte, and U. V. Waghmare, Phase Transitions: A Multinational Journal 79, 91 (2006).

${ }^{35}$ P. Ganesh, E. Cockayne, M. Ahart, R. E. Cohen, B. Burton, R. J. Hemley, Y. Ren, W. Yang, and Z.-G. Ye, Phys. Rev. B 81, 144102 (2010).

${ }^{36}$ M. Sepliarsky, A. Asthagiri, S. Phillpot, M. Stachiotti, and R. Migoni, Curr. Opin. Solid State Mater. Sci. 9, 107 (2005).

${ }^{37}$ M. Sepliarsky and R. E. Cohen, J. Phys.: Condens. Matter 23, 435902 (2011).

${ }^{38}$ H. Luo, G. Xu, H. Xu, P. Wang, and Z. Yin, Jpn. J. Appl. Phys. 39, 5581 (2000).

${ }^{39}$ A. Al-Zein, B. Hehlen, J. Rouquette, and J. Hlinka, Phys. Rev. B 78, 134113 (2008).

${ }^{40}$ A. Al-Zein, J. Hlinka, J. Rouquette, and B. Hehlen, Phys. Rev. Lett. 105, 017601 (2010).

${ }^{41}$ I. P. Swainson, C. Stock, P. M. Gehring, G. Xu, K. Hirota, Y. Qiu, H. Luo, X. Zhao, J.-F. Li, and D. Viehland, Phys. Rev. B 79, 224301 (2009).

${ }^{42}$ S. B. Vakhrushev, B. E. Kvyatkovsky, A. A. Naberezhnov, N. M. Okuneva, and B. P. Toperverg, Ferroelectrics 90, 173 (1989).

${ }^{43}$ K. Z. Baba-Kishi and D. J. Barber, J. Appl. Crystallogr. 23, 43 (1990).

${ }^{44}$ M. A. Akbas and P. K. Davies, J. Am. Ceram. Soc. 80, 2933 (1997).

${ }^{45}$ Y. Yan, S. J. Pennycook, Z. Xu, and D. Viehland, Appl. Phys. Lett. 72, 3145 (1998).

${ }^{46}$ Q. Zhang, H. You, M. L. Mulvihill, and S. Jang, Solid State Commun. 97, 693 (1996).

${ }^{47}$ L. Bursill, P. JuLin, Q. Hua, and N. Setter, Phys. B (Amsterdam, Neth.) 205, 305 (1995).
${ }^{48}$ L. Bursill, H. Qian, J. Peng, and X. Fan, Phys. B (Amsterdam, Neth.) 216, 1 (1995).

${ }^{49}$ M. Sepliarsky, Z. Wu, A. Asthagiri, and R. Cohen, Ferroelectrics 301, 55 (2004).

${ }^{50}$ M. Ahart, A. Asthagiri, Z.-G. Ye, P. Dera, H.-K. Mao, R. E. Cohen, and R. J. Hemley, Phys. Rev. B 75, 144410 (2007).

${ }^{51}$ P. Bonneau, P. Garnier, E. Husson, and A. Morell, Mater. Res. Bull. 24, 201 (1989).

${ }^{52}$ S. A. Prosandeev, E. Cockayne, B. P. Burton, S. Kamba, J. Petzelt, Y. Yuzyuk, R. S. Katiyar, and S. B. Vakhrushev, Phys. Rev. B 70, 134110 (2004).

${ }^{53}$ I. Etxebarria, J. M. Perez-Mato, A. García, P. Blaha, K. Schwarz, and J. Rodriguez-Carvajal, Phys. Rev. B 72, 174108 (2005).

${ }^{54}$ J. D. Gale and A. L. Rohl, Mol. Simul. 29, 291 (2003).

${ }^{55}$ I. T. Todorov, W. Smith, K. Trachenko, and M. T. Dove, J. Mater. Chem. 16, 1911 (2006).

${ }^{56}$ T. Proffen and R. B. Neder, J. Appl. Crystallogr. 30, 171 (1997).

${ }^{57}$ T. R. Welberry, R. L. Withers, and S. C. Mayo, J. Solid State Chem. 115, 43 (1995).

${ }^{58}$ T. R. Welberry, J. Appl. Crystallogr. 19, 382 (1986).

${ }^{59}$ C. Stock, L. Van Eijck, P. Fouquet, M. Maccarini, P. M. Gehring, G. Xu, H. Luo, X. Zhao, J.-F. Li, and D. Viehland, Phys. Rev. B 81, 144127 (2010)

${ }^{60}$ J. Hlinka, J. Petzelt, S. Kamba, D. Noujni, and T. Ostapchuk, Phase Transitions 79, 41 (2006).

${ }^{61}$ V. Bobnar, C. Filipič, A. Levstik, and Z. Kutnjak, J. Appl. Phys. 107, 084104 (2010).

${ }^{62}$ G. Xu, Z. Zhong, Y. Bing, Z.-G. Ye, and G. Shirane, Nature Mater. 5, 134 (2006). 